\title{
Weak lensing constraints on the dark matter haloes of early type galaxies
}

\author{
Priyamvada Natarajan ${ }^{1,2}$ \\ ${ }^{1}$ Radcliffe Institute for Advanced Study, 8 Garden Street, Cambridge, MA 02138, USA \\ ${ }^{2}$ Dept. of Astronomy, Yale University, 260 Whitney Avenue, New Haven, CT 06520, USA \\ email: priyamvada.natarajan@yale.edu
}

\begin{abstract}
Constraints on the masses of dark matter haloes associated with galaxies in the field and in clusters have been successfully obtained used galaxy-galaxy lensing techniques. Weak lensing thus provides important information on the mass distribution in galaxies at large radii in a statistical fashion. Current work suggests that all galaxies possess extended dark matter haloes, however those that host galaxies in clusters are less extended and less massive.
\end{abstract}

Keywords. dark matter, galaxies

\section{Introduction}

Gravitational lensing has emerged as one of the most powerful techniques to map mass distributions on a range of scales: galaxies, clusters and beyond. The distortion in the shapes of background galaxies viewed through fore-ground mass distributions is independent of the dynamical state of the lens, therefore, unlike other methods for mass estimation there are fewer biases in lensing determinations. Here, we focus on the statistical mass estimates of field and cluster early-type galaxies obtained using weak gravitational lensing. Galaxy-galaxy lensing, the preferential alignment of faint background galaxies around bright foreground galaxies, was originally proposed as a method to constrain the masses and spatial extents of field galaxies (Brainerd, Blandford \& Smail 1996), but has since been very successfully extended and applied inside clusters (Natarajan \& Kneib 1996; Natarajan et al. 1998; 2002a). Constraints from this technique are statistical and probe the mass profiles at large radii, typically $r>0.1 r_{v i r}$. One advantage of probing this scale in galaxies is that uncertainties due to baryonic effects do not dominate, unlike measurements on smaller scales.

\section{Galaxy-galaxy lensing constraints on field galaxies}

Early results on galaxy-galaxy lensing identified a signal associated with massive haloes around typical early-type field galaxies, extending to beyond $100 \mathrm{kpc} \dagger$ (e.g. Brainerd, Blandford \& Smail 1996; Hudson et al. 1998; Hoekstra et al. 2004). In particular, Hoekstra et al. (2004) report the detection of finite truncation radii based on the imaging data from the Red-Sequence Cluster Survey. Using a truncated isothermal sphere to model the mass in galaxy haloes, they find a best-fit central velocity dispersion for an $L^{*}$ galaxy of $\sigma=$ $136 \pm 5 \mathrm{kms}^{-1}$ (68\% confidence limits) and a truncation radius of $185 \pm 30 \mathrm{kpc}$. However,

$\dagger$ We adopt $\mathrm{h}=\mathrm{H}_{o} / 100 \mathrm{~km} \mathrm{~s}^{-1} \mathrm{Mpc}^{-1}=0.7$ and $\Omega_{\Lambda}=0.7$. 
the most exciting results on the masses, density profiles and the galaxy-mass correlation function at the present time are from the SDSS (Sloan Digital Sky Survey). Mandelbaum and collaborators (Mandelbaum et al. 2006; Hirata et al. 2007) find that mass traces light on these large scales as $M / L \sim L^{0.5}$; the mass of a typical dark matter (DM) halo that hosts an early-type $\mathrm{L}^{*}$ galaxy is $1.5 \times 10^{12} h^{-1} M_{\odot}$; more massive $\mathrm{DM}$ haloes host brighter galaxies and that the masses of haloes that host late-types are a factor of 2-4 lower. Fitting parametric models they find that both NFW and Einasto profiles offer good fits over the range $0.2 r_{v i r}<r 1 h^{-1} \mathrm{Mpc}$; and the inferred concentration parameter $c_{v i r}$ for NFW profiles is consistent with expectations of a power-law distribution with halo mass from cosmological simulations.

\section{Galaxy-galaxy lensing constraints on cluster galaxies}

Isolating the galaxy-galaxy lensing signal in clusters has been a fruitful enterprise with HST (Hubble Space Telescope) quality data. Constraints have been obtained on the host haloes for fiducial early-types in $\sim 10$ clusters. DM haloes in the cluster environment appear to be severely truncated compared to their field counterparts. Although there is some variation amongst clusters, the typical mass of a DM halo that hosts an $\mathrm{L}^{*}$ galaxy in clusters is a factor of 2-5 lower than an equivalent field galaxy. In addition, to the fact that tidal truncation in the violent cluster environment appears to strip infalling DM haloes, a strong radial trend with projected cluster-centric distance has also been detected for the cluster $\mathrm{Cl0024+16}$ (Natarajan et al. 2009). Utilizing mosaic-ed HST data extending well beyond the virial radius for the cluster $\mathrm{Cl} 0024+16$, we find that fiducial DM haloes are more massive the further away they are from the cluster center, as expected from dynamics and crossing times (Treu et al. 2003). A first detection of the DM halo associated with late-type galaxies is also reported in this cluster. The DM halo hosting a late-type appears to be more severely tidally stripped than the equivalent halo hosting an early-type in the same cluster environment. Comparing with high resolution cosmological simulations, it is found that the observationally detected halo sizes and masses are consistent within the errors with expectations in $\Lambda$ CDM paradigm (Natarajan \& Springel 2004; Natarajan, De Lucia \& Springel 2007).

\section{Conclusions}

Current weak lensing observations and analysis provide evidence for the existence of extended DM haloes around most galaxies. The DM haloes associated with earlytype galaxies both in the field and in clusters are more massive than the haloes hosting equivalent luminosity late-types. These results offer deeper insights into the interplay between baryons and dark matter as a function of environment. Future large surveys are likely to help disentangle this relationship further.

\section{References}

Brainerd, T., Blandford, R., \& Smail, I., 1996, ApJ, 466, 623

Hirata, C., et al., 2007, MNRAS, 381, 1197

Hoekstra, H., Yee, H. K., \& Gladders, M., 2004, ApJ, 606, 67

Hudson, M. J., Gwyn, S. D. J., Dahle, H., \& Kaiser, N., 1998, ApJ, 503, 531

Mandelbaum, R., Seljak, U., Kauffmann, G., Hirata, C., \& Brinkmann, J.,, 2006, MNRAS, 368, 715 
Natarajan, P. \& Kneib, J.-P. 1997, MNRAS, 287, 833

Natarajan, P., Kneib, J.-P., Smail, I., \& Ellis, R. S. 1998, ApJ, 499, 600

Natarajan, P., Kneib, J.-P., \& Smail, I., 2002a, ApJ, 580, L11

Natarajan, P. \& Springel, V., 2004, ApJ Lett., 617, 13

Natarajan, P., De Lucia, G., \& Springel, V., 2007, MNRAS, 376, 180

Natarajan, P., et al., 2009, ApJ, 693, 970

Treu, T., et al., 2003, ApJ, 591, 53 